\title{
Antibiotic use for pneumonia among children under-five at a pediatric hospital in Dhaka city, Bangladesh
}

This article was published in the following Dove Press journal:

Patient Preference and Adherence

3 August 2017

Number of times this article has been viewed

\author{
Md Mahbubur Rashid ${ }^{1,2}$ \\ Mohammod Jobayer Chisti ${ }^{3}$ \\ Dilruba Akter ${ }^{4}$ \\ Malabika Sarkar ${ }^{2}$ \\ Fahmida Chowdhury' \\ 'Infectious Diseases Division, \\ International Centre for Diarrhoeal \\ Disease Research, Bangladesh \\ (icddr,b), Dhaka, Bangladesh; 'James \\ P Grant School of Public Health, \\ BRAC University, Dhaka, Bangladesh; \\ ${ }^{3}$ Nutrition and Clinical Services \\ Division, International Centre \\ for Diarrhoeal Disease Research, \\ Bangladesh (icddr,b), Dhaka, \\ Bangladesh; ${ }^{4}$ Department of Pathology, \\ Shaheed Tajuddin Ahmed Medical \\ College Hospital, Gazipur, Dhaka, \\ Bangladesh
}

Background: Pneumonia has been the leading cause of morbidity and mortality among children under 5 for more than 3 decades, particularly in low-income countries like Bangladesh. The World Health Organization (WHO) developed a pneumonia case management strategy which included the use of antibiotics for both primary and hospital-based care. This study aims to describe antibiotic usage for treating pneumonia in children in a private pediatric teaching hospital in Dhaka, Bangladesh.

Methods: We conducted this cross-sectional study among children $<5$ years old who were admitted to a private pediatric hospital in Dhaka with a diagnosis of pneumonia in November 2012.

Results: We enrolled 80 children during the study period. Among them, 28 (35.4\%) were underweight, 14 (17.7\%) were moderately underweight, and $13(16.5 \%)$ were severely underweight. On the basis of WHO classification (2005), 43 children (54\%) had severe pneumonia and 37 (46\%) had very severe pneumonia, as diagnosed by the research physician. Among the prescribed antibiotics in the hospital, parenteral ceftriaxone was the most common $40(50 \%)$, followed by cefotaxime plus amikacin 14 (17.5\%), cefuroxime 7 (8.8\%), ceftazidime plus amikacin $6(7.5 \%)$, ceftriaxone plus amikacin $3(3.8 \%)$, meropenem $2(2.5 \%)$, cefepime 2 $(2.5 \%)$, and cefotaxime $2(2.5 \%)$.

Conclusion: Despite the WHO pneumonia treatment strategy, the inappropriate use of highergeneration cephalosporin and carbapenem was high in the study hospital. The results underscore the noncompliance with the WHO guidelines of antibiotic use and the importance of enforcing regulatory policy of the rational use of antibiotics for treating hospitalized children with pneumonia. Following these guidelines may help prevent increased antimicrobial resistance.

Keywords: antibiotic use, under-five children, pneumonia, private hospitals, pediatrics, WHO guidelines, Bangladesh

\section{Introduction}

Globally, pneumonia is one of the leading causes of morbidity and mortality and accounted for $16 \%$ of the estimated 5.9 million deaths among children aged $<5$ years in 2015. ${ }^{1}$ In 2010, there were 120 million episodes of pneumonia in children younger than 5 years, and 14 million pneumonia cases progressed to severe and life-threatening conditions requiring urgent hospital care. ${ }^{2}$ In 2011, about 1.3 million estimated pneumonia cases led to death worldwide. ${ }^{2}$ Pneumonia is one of the leading causes for hospital admission among children under 5 years of age in pediatric hospitals in Bangladesh. ${ }^{3}$ Among the estimated 119,000 under-five deaths in Bangladesh, 15\% were due to pneumonia. ${ }^{1}$
Correspondence: Fahmida Chowdhury Respiratory Viruses Working Group, Programme on Emerging infections, Infectious Diseases Division,

icddr,b, Bangladesh

Tel +l 880181705448 I

Email fahmida_chow@icddrb.org 
In 1980, the World Health Organization (WHO) developed a case-management strategy known as the Acute Respiratory Infection (ARI) program in an attempt to reduce pneumonia-related deaths. Thereafter, in 1995, this strategy was incorporated into the guidelines of the Integrated Management of Childhood Illness (IMCI) for primary care and hospital-based case management. In 2005, the IMCI guidelines were incorporated in the WHO Pocket Book for pediatric hospital care with some amendments, especially for physicians. The impact of these guidelines has been enormous. Over the decades, death from pneumonia in children under five dropped from 2.3 million in 1990 to 0.9 million in 2015. ${ }^{1}$ However, studies in many countries have reported that health workers are not adhering to the IMCI guidelines in disease diagnosis, antibiotic prescription, and referrals. ${ }^{4-7}$ In addition, there is a scarcity of research from referral hospitals that assess adherence to the IMCI guidelines. ${ }^{6}$ Before 2013, the IMCI guidelines were based on low-quality evidence and that might have had an impact on antibiotic use among clinicians at referral centers. In 2013, the WHO revised the classification of pneumonia and the treatment regimen on the basis of high-quality up-to-date evidence, aiming to improve the quality of care and reduce deaths especially in resource-poor settings. Additional aims of the revision were to increase utilization of the guidelines by physicians, nurses, and senior health workers responsible for treating young children at primary health care centers. ${ }^{8}$

Antibiotics are the most frequently prescribed medicament for acute respiratory tract infections and acute watery diarrhea. ${ }^{9,10}$ According to the Bangladesh Demographic and Health Survey (2013), 71.4\% of under-five children received antibiotics for ARI or pneumonia from a health facility or treatment provider. ${ }^{11}$ A study conducted in the south-west of Bangladesh showed that cephalosporins accounted for $31.8 \%$ of total antibiotic prescriptions, and among them, cefixime, cefuroxime, and ceftriaxone were highly used for respiratory and other infections and $83 \%$ prescriptions were used irrationally without any positive microbial test. ${ }^{10}$ According to the WHO, common health care-associated and communityacquired pneumonia are now caused by highly resistant bacteria in all WHO regions. ${ }^{12}$ In Bangladesh, studies have shown antibiotics use on pediatric and adult patients at a medical university and regional health complexes for a variety of diseases, including ARI or pneumonia. ${ }^{13-15}$ However, there remains scarce information regarding antibiotic practices in cases of severe and very severe pneumonia among children under 5 years in private hospitals. In this study, we aimed to describe antibiotic practice for treating pneumonia among children under 5 years of age in a private pediatric teaching hospital in Dhaka, Bangladesh.

\section{Methodology}

From 13th to 23rd of November 2012, we conducted a crosssectional study among children under 5 years of age admitted with a diagnosis of pneumonia at a private pediatric teaching hospital in Dhaka.

Using a $95 \% \mathrm{CI}$ and $10 \%$ precision, we assumed that $71 \%$ of pneumonia patients would receive first-line treatment with antibiotics ${ }^{11}$ and calculated a total sample size of 80 .

Children of either sex, under 5 years of age with a clinical diagnosis of pneumonia according to WHO Pocket Book $(2005)^{16}$ irrespective of pneumonia etiology, admitted within the given time period in the study hospital, were enrolled after having informed consent of the parents. Children with comorbidities such as congenital heart disease, meningitis, and chronic respiratory diseases such as chronic suppurative lung disease and tuberculosis reported in the patient history form were excluded. We followed WHO criteria ${ }^{16}$ to develop the operational definitions of childhood pneumonia.

\section{Pneumonia}

The case definition of pneumonia included children presenting with cough and difficulty in respiration having age-specific fast breathing ( $\geq 50$ breaths/minute for 2 to $<12$ months of age and $\geq 40$ breaths/minute for $12-59$ months of age). Fever $\left(\mathrm{T} \geq 38.0^{\circ} \mathrm{C}\right)$ may or may not be present for diagnosis.

\section{Severe pneumonia}

The case definition of severe pneumonia included children presenting with cough and difficulty in respiration having $\geq 60$ breaths/minute and/or lower chest wall in-drawing ( 0 to $<2$ months), and lower chest wall indrawing irrespective of the presence or absence of the agespecific fast breathing.

\section{Very severe pneumonia}

Very severe pneumonia included patients with cough or difficulty breathing with any of the following danger signs - central cyanosis, inability to breastfeed or drink, or uncontrollable vomiting, convulsion, lethargy or unconsciousness, or severe respiratory distress (eg, head nodding).

\section{Data collection instruments and tools}

A pretested structured questionnaire was used to collect demographic information, monthly income status, clinical 
presentation, physical findings, immunization history, exclusive breast feeding history, nutritional status, past medical history, history of antibiotic use prior to hospitalization, treatment providers prior to hospitalization, primary diagnosis, additional diagnosis or comorbidities diagnosed by the hospital's physicians, diagnosis made by the researcher, and antibiotic prescription after hospitalization. Hospital registration number, primary diagnosis, additional diagnosis or comorbidities reported by the hospital's physicians, and the treatment prescribed during hospitalization were collected from the participants' history form at the hospital. Additional diagnosis or comorbidities included febrile convulsion, septicemia (seriously ill with no apparent cause, purpura, petechiae, shock, and hypothermic young infant or severely malnourished child), bronchial asthma (recurrent episodes of shortness of breath or wheeze), enteric fever (fever with any of the following: diarrhea or constipation, vomiting, abdominal pain, headache, and cough, particularly if the fever has persisted for $\geq 7$ days), ${ }^{16}$ acute gastroenteritis, childhood obesity, ${ }^{17}$ and cerebral palsy.

Stethoscope, blood pressure machine, timer, length/height scale, weight scale, mid upper arm circumference tape, and digital thermometer were used for data collection.

\section{Data management and statistical analysis}

According to the WHO Pocket Book (2005), ${ }^{16}$ we stratified the children into three age groups for diagnostic purposes. $Z$ scores were calculated using the new child growth standards of the WHO. ${ }^{18,19}$ Children with a $Z$ score for their median weight $>-1$ were considered as not underweight, -1 to $>-2$ as underweight, -2 to $>-3$ as moderately underweight, and $<-3$ as severely underweight.

The data were entered using SPSS 20 and analyzed using STATA 12 . To calculate the weight-for-age $Z$ score, WHO Anthro software v3.2.2 was used. Continuous variables were reported by the mean value or median value and range. Pearson's chi-squared test was used for bivariate analysis to show the association between antibiotic use, age of patients, clinical findings, chief complaints, and comorbidities.

\section{Ethical consideration}

Data were collected from each participant after receiving informed written consent by explaining explicitly the objectives and procedures of the study to the parents or the caregiver of the participants. The study was approved by the Ethical Review Committee of James P Grant School of Public Health, BRAC University.

\section{Findings}

We enrolled 80 children from the study hospital during the given time period. Most of the participants were male (72.5\%) and $41 \%$ were aged between 3 and 12 months (Table 1). Thirty percent of the children were not underweight, $41 \%$ were exclusively breastfed, and $53.7 \%$ received their vaccinations according to the expanded program on immunization schedule. The monthly household income was $<188$ USD ( 1 USD $=80$ Bangladeshi taka) for $47.5 \%$ participants, $86.4 \%$ of the children's primary caregivers were their mothers, and $28.7 \%$ of the participants were admitted from outside of Dhaka (Table 1).

The study participants $(80 ; 100 \%)$ were diagnosed as pneumonia by the hospital's physicians. However, according

Table I Sociodemographic characteristics of hospitalized pneumonia children in a private pediatric hospital in Dhaka, Bangladesh, November, 2012

\begin{tabular}{|c|c|}
\hline Variables $(\mathbf{N}=\mathbf{8 0})$ & n (\%) \\
\hline \multicolumn{2}{|c|}{ Age of participants according to WHO classification for } \\
\hline \multicolumn{2}{|l|}{ Pneumonia, months } \\
\hline $0-2$ & $18(22.5)$ \\
\hline $3-12$ & $33(41.3)$ \\
\hline $13-59$ & $29(36.3)$ \\
\hline Age of participants in months* median (IQR) & $8.5(3-16)$ \\
\hline \multicolumn{2}{|l|}{ Sex } \\
\hline Male & $58(72.5)$ \\
\hline Female & $22(27.5)$ \\
\hline \multicolumn{2}{|l|}{ Nutritional status $(n=79)^{a}$} \\
\hline Not underweight & $24(30.3)$ \\
\hline Underweight & $28(35.4)$ \\
\hline Moderately underweight & $14(17.7)$ \\
\hline Severely underweight & $13(16.5)$ \\
\hline \multicolumn{2}{|l|}{ Monthly income of parents of the participants, BDT } \\
\hline$<15,000$ & $38(47.5)$ \\
\hline $15-25,000$ & $22(27.5)$ \\
\hline$>25,000$ & $20(25.0)$ \\
\hline \multicolumn{2}{|l|}{ Caregivers of participants } \\
\hline Mothers & $69(86.4)$ \\
\hline Fathers & $10(12.5)$ \\
\hline Others & $\mathrm{I}(\mathrm{I} .3)$ \\
\hline \multicolumn{2}{|l|}{ Residence of participants } \\
\hline Dhaka & $57(7 \mathrm{I} .3)$ \\
\hline Outside of Dhaka & $23(28.7)$ \\
\hline Vaccination $(n=67)^{b}$ & $67(83.7)$ \\
\hline \multicolumn{2}{|l|}{ Status of vaccination $(n=67)^{b}$} \\
\hline Complete & $36(53.7)$ \\
\hline Age appropriate & $31(46.3)$ \\
\hline Exclusive breast feeding & $33(41.3)$ \\
\hline $\begin{array}{l}\text { Exclusive breast feeding in months }(\mathrm{n}=33)^{*} \\
\text { median (IQR) }\end{array}$ & $3(2-6)$ \\
\hline
\end{tabular}

Notes: *Median and interquartile range (IQR) are mentioned for continuous variable. ${ }^{a}$ For one participant, weight-for-age $Z$ score cannot be calculated as the weight was extremely high according to age. 'Status of vaccination among vaccinated children.

Abbreviation: BDT, Bangladeshi taka. 
to the WHO classification, ${ }^{16} 43(53.8 \%)$ were diagnosed as severe pneumonia and $37(46.3 \%)$ as very severe pneumonia by the study physician.

Among the study participants, 77 (96.3\%) suffered from cough, 77 (96.3\%) from breathlessness and fast breathing, $71(88.8 \%)$ from a loss of appetite, 55 (68.8\%) from fever, $54(67.5 \%)$ from runny nose, $34(42.5 \%)$ from vomiting, and $8(10.1 \%)$ from convulsion. The median duration of the runny nose was 5 days (interquartile range $[\mathrm{IQR}]=3-7$ ), cough was 5 days (IQR $=3-7$ ), breathlessness and fast breathing was 3 days ( $\mathrm{IQR}=2-4)$, loss of appetite was 3 days ( $\mathrm{IQR}=2-4$ ), fever was 2 days (IQR $=1-4)$, vomiting was 2 days $(\mathrm{IQR}=1-3)$, and convulsion was 1 day $(\mathrm{IQR}=1-3)$. Among the participants, $5(6.3 \%)$ had associated comorbidities including febrile convulsion, 2 (2.5\%) septicemia, $2(2.5 \%)$ acute gastroenteritis, 1 (1.3\%) bronchial asthma, $1(1.3 \%)$ childhood obesity, 1 (1.3\%) cerebral palsy, and $1(1.3 \%)$ enteric fever.

After hospital admission, both single and a combination of double antibiotics were used (Figure 1). In most of the cases, third-generation cephalosporin was prescribed. Fifty-eight (72.5\%) participants were admitted for the first time in the hospital in their life time. Fifty-eight $(72.5 \%)$ participants received treatment from physicians and $1(1.3 \%)$ from a drug seller in a pharmacy before hospitalization. Among them, 37 (46.3\%) were prescribed antibiotics and the median duration of antibiotic use prior to admission was 2 days (IQR $=1-3$ ). A wide range of oral to injectable antibiotics were used by the participants prior to hospital admission (Figure 2).

For both severe and very severe pneumonia, ceftriaxone was the most commonly prescribed antibiotic after hospitalization (Figure 3). Cefuroxime was prescribed more often for severe pneumonia patients when compared with those who had very severe pneumonia. Ceftazidime, cefotaxime plus gentamycin, cefepime, and ampicillin plus gentamycin were prescribed only to $5(13.5 \%)$ participants diagnosed as very severe pneumonia (Figure 3 ).

Among the prescribed antibiotics, ceftriaxone $(P<0.001)$ and the combination of cefotaxime and amikacin $(P=0.01)$ were found to be significantly associated with the age of the participants (Table 2). Ceftazidime plus amikacin was associated with auscultatory crepitation in lungs $(P=0.04)$. Cefuroxime was associated with the chief complaints of breathlessness, cold, and crying $(P=0.01)$ and patients having no other comorbidity $(P=0.01)$ (Table 2$)$.

\section{Discussion}

The most important observation of this study was the lack of adherence to WHO guidelines for diagnosing and treating pneumonia by the hospital's physicians. All children admitted with severe pneumonia and very severe

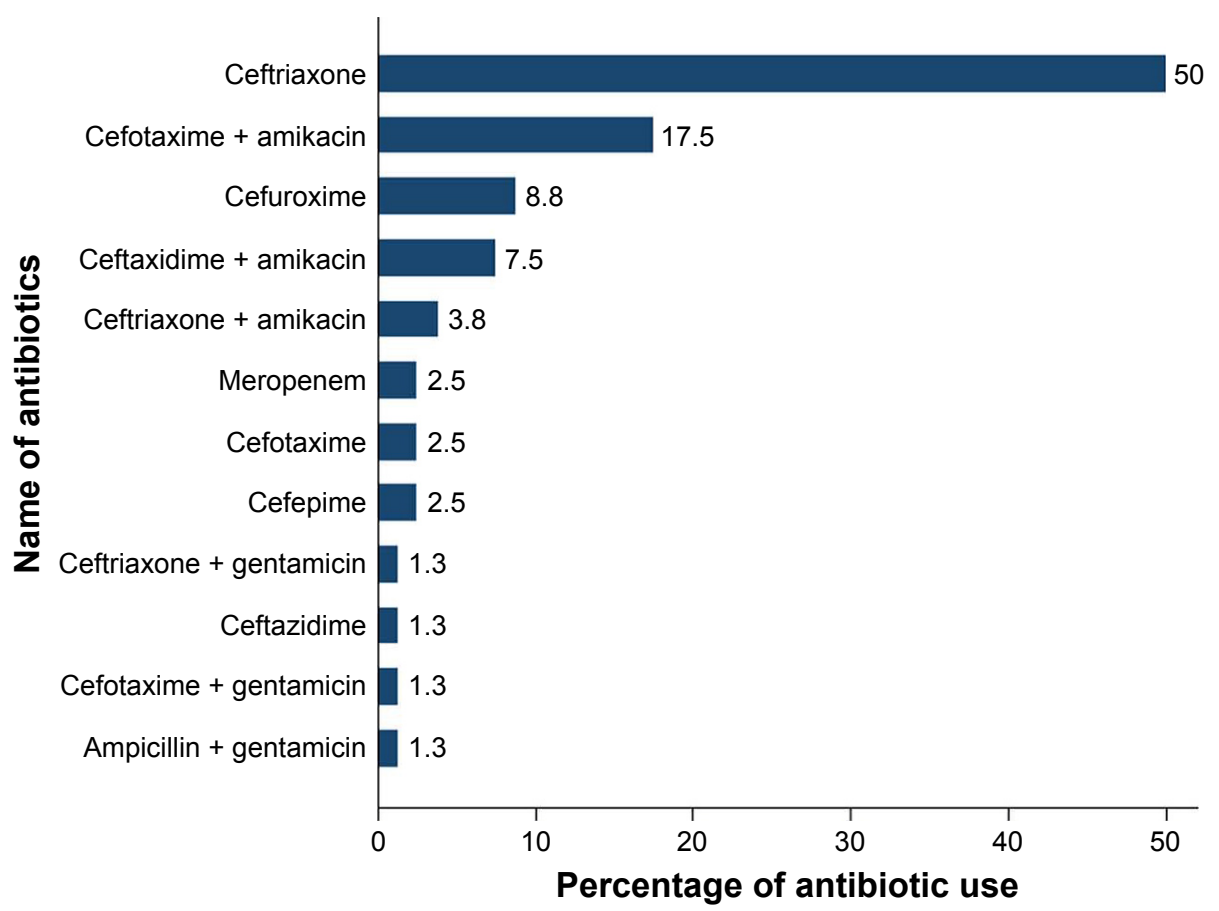

Figure I Use of antibiotics by the hospital physicians among the hospitalized pneumonia children in a private pediatric teaching hospital in Dhaka, Bangladesh, November, 2012. 


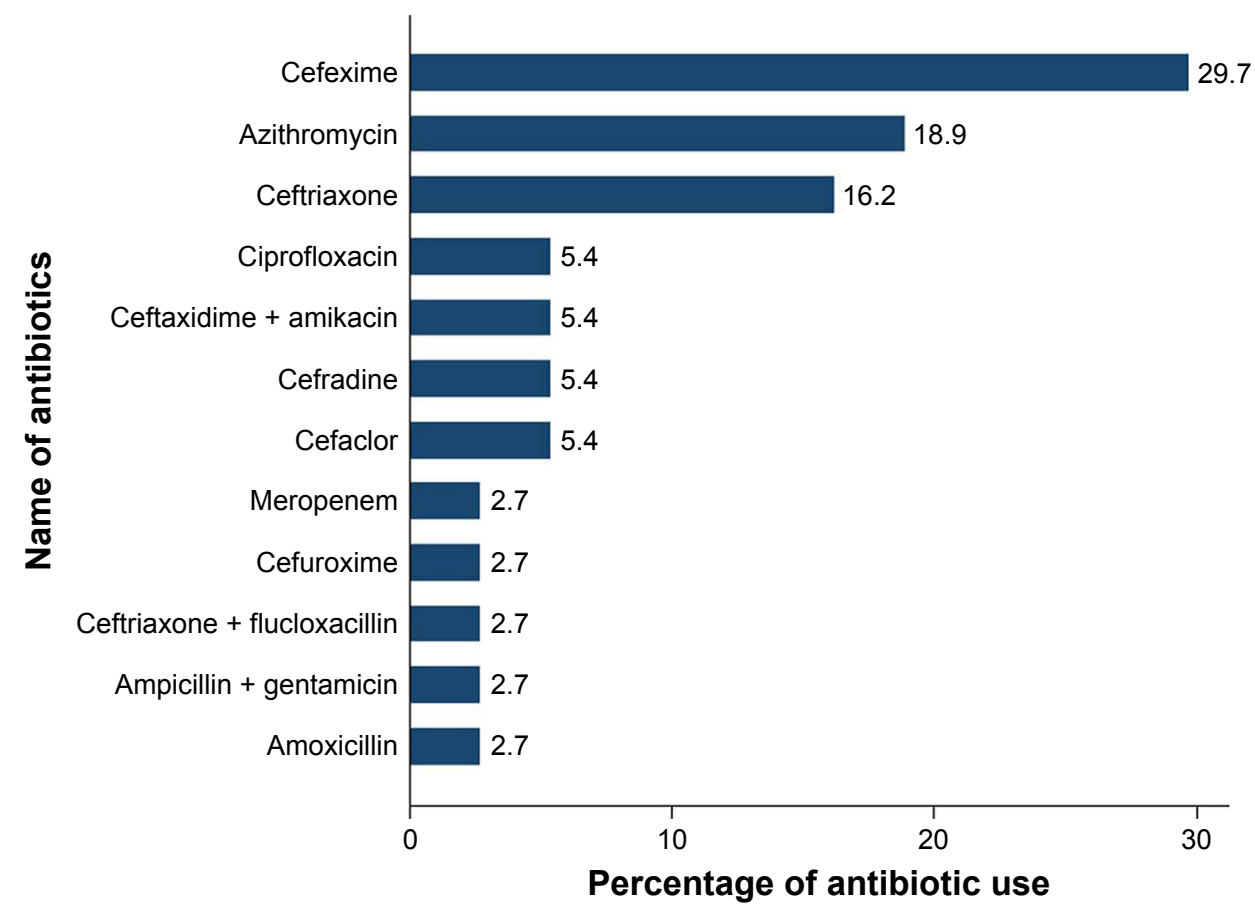

Figure 2 Antibiotics used before hospitalization among the hospitalized pneumonia children in a private pediatric teaching hospital in Dhaka, Bangladesh, November, 2012.

pneumonia received a wide range of extended spectrum antibiotics through a parenteral route. A study conducted in 10 upazila health complexes in Dhaka and Noakhali district of Bangladesh also showed that injectable antibiotics were used in $100 \%$ of $\mathrm{ARI} /$ pneumonia encounters at 4 out of 5 upazila health complexes in Dhaka District. ${ }^{20}$ According to Akter et al, ${ }^{21}$ the commonly used antibiotics in Bangladesh were ampicillin, gentamicin, amoxicillin, cloxacillin, and

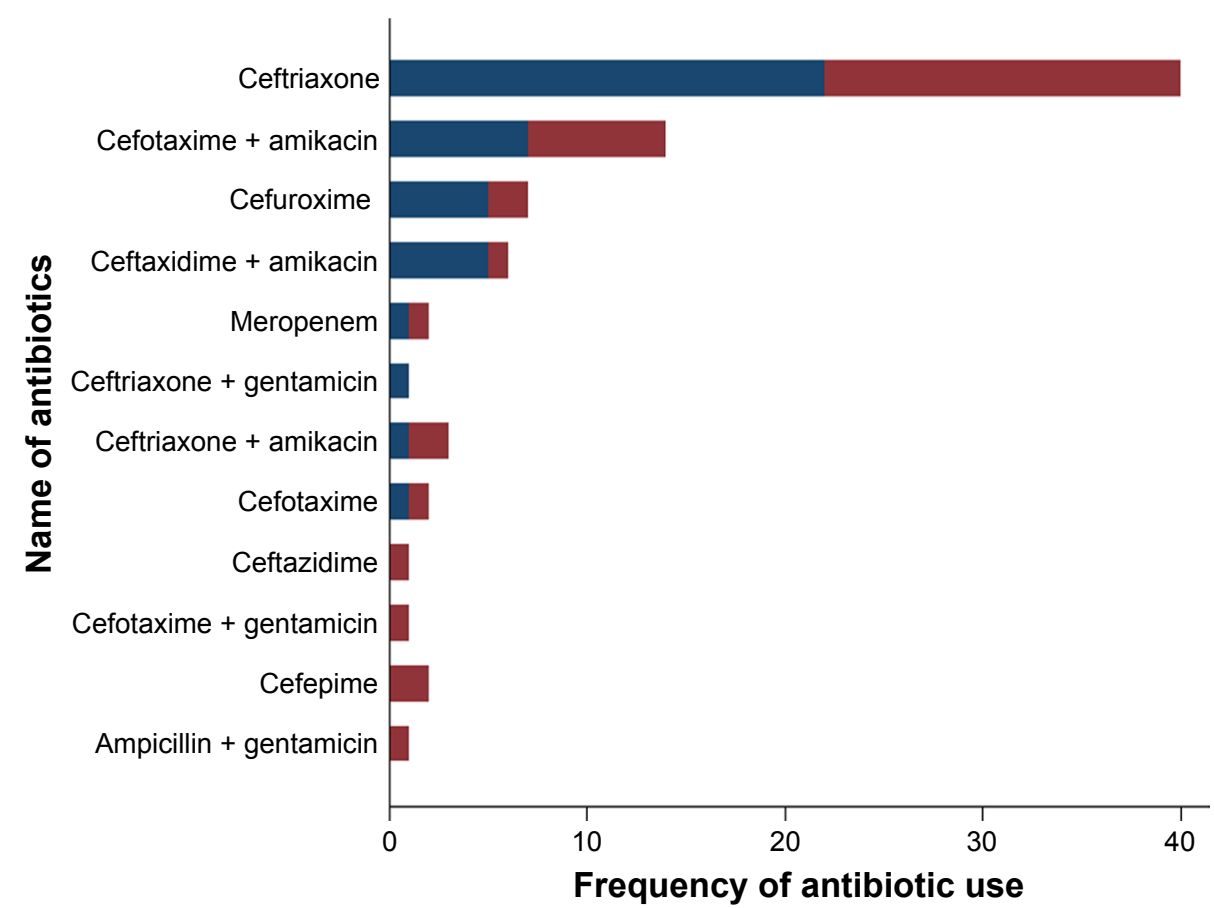

Severe pneumonia Very severe pneumonia

Figure 3 Difference in using antibiotics for treating severe pneumonia and very severe pneumonia in hospital among the hospitalized pneumonia pediatric patients in a private pediatric teaching Hospital in Dhaka, Bangladesh, November, 2012. 
Table 2 Association of antibiotic use with demographic information, history of illness, and clinical findings of the patients admitted with pneumonia in a private pediatric teaching hospital in Dhaka, Bangladesh, November, 2012

\begin{tabular}{|c|c|c|c|}
\hline $\begin{array}{l}\text { Name of } \\
\text { antibiotics }\end{array}$ & $\begin{array}{l}\text { Variable regarding } \\
\text { demographic information, } \\
\text { history of illness, and } \\
\text { clinical findings }\end{array}$ & $\begin{array}{l}\text { Use of } \\
\text { different } \\
\text { antibiotics } \\
\text { n (\%) }\end{array}$ & $P$ trend* \\
\hline \multirow[t]{4}{*}{ Ceftriaxone } & \multicolumn{3}{|l|}{ Age of the children } \\
\hline & $0-2$ months & $\mathrm{I}(2.5)$ & \\
\hline & $3-12$ months & $15(37.5)$ & $<0.001$ \\
\hline & $13-59$ months & $24(60.0)$ & \\
\hline \multirow{4}{*}{$\begin{array}{l}\text { Cefotaxime + } \\
\text { amikacin }\end{array}$} & \multicolumn{3}{|l|}{ Age of the children } \\
\hline & $0-2$ months & $5(35.7)$ & \\
\hline & $3-12$ months & $9(64.3)$ & \multirow[t]{2}{*}{0.01} \\
\hline & $13-59$ months & $0(0.0)$ & \\
\hline \multirow{4}{*}{$\begin{array}{l}\text { Ceftazidime + } \\
\text { amikacin }\end{array}$} & \multicolumn{3}{|c|}{ Respiratory auscultation findings } \\
\hline & Crepitation & $6(100.0)$ & \multirow[t]{3}{*}{0.04} \\
\hline & Rhonchi & $0(0.0)$ & \\
\hline & Crepitation with rhonchi & $0(0.0)$ & \\
\hline \multirow[t]{18}{*}{ Cefuroxime } & \multicolumn{3}{|c|}{ Chief complaints reported by guardians } \\
\hline & Breathlessness & $4(57.1)$ & \\
\hline & Cold & $2(28.6)$ & \\
\hline & Crying & I (14.3) & \\
\hline & Cough & $0(0.0)$ & 0.01 \\
\hline & Fast breathing & $0(0.0)$ & \\
\hline & Fever & $0(0.0)$ & \\
\hline & Reluctant to take food & $0(0.0)$ & \\
\hline & Chest indrawing & $0(0.0)$ & \\
\hline & \multicolumn{3}{|l|}{ Comorbidities } \\
\hline & No other disease & $4(57.1)$ & \\
\hline & Childhood obesity & I (14.3) & \\
\hline & Cerebral palsy & I (14.3) & \\
\hline & Febrile convulsion & I (14.3) & 0.01 \\
\hline & Acute gastroenteritis & $0(0.0)$ & \\
\hline & Enteric fever & $0(0.0)$ & \\
\hline & Septicemia & $0(0.0)$ & \\
\hline & Asthma & $0(0.0)$ & \\
\hline
\end{tabular}

Notes: *Pearson's chi-square was used for bivariate analysis. The result is not significant at $P>0.05$.

ceftriaxone for treating pneumonia and diarrhea for all the pediatric age groups; however adherence to WHO guidelines was not evaluated in the study. At a tertiary hospital in Malaysia , the commonly used antibiotics were amoxicillin with clavulanate (augmentin) followed by erythromycin, cefuroxime, ceftriaxone, and ampicillin with sulbactum sodium, irrespective of the patients' age group, which may lead to inappropriate prescription of antibiotics and which might be influenced by physicians' personal choice and limited experience. ${ }^{22}$ Our findings showed that ceftriaxone, cefotaxime, amikacin, cefuroxime, and ceftazidime were commonly used for the treatment of pneumonia after hospital admission. Among the severe pneumonia cases,
$67.4 \%$ were treated with third-generation cephalosporin and second-generation cephalosporin, carbapenem, and $32.6 \%$ cases were treated with a combination of two antibiotics such as cefotaxime plus amikacin, ceftazidime plus amikacin, ceftriaxone plus gentamicin, and ceftriaxone plus amikacin. On the contrary, among the very severe pneumonia cases, $67.6 \%$ were treated with fourth-generation cephalosporin, third-generation cephalosporin, and secondgeneration cephalosporin, carbapenem, and 29.7\% were treated with a combination of two drugs such as cefotaxime plus amikacin, ceftriaxone plus amikacin, ceftaxidime plus amikacin, cefotaxime plus gentamicin, and ceftriaxone plus gentamicin other than ampicillin plus gentamicin. The use of antibiotics during admission was high compared to the results of a previous study conducted by Akter et a ${ }^{21}$ in Bangladesh. It also appeared that physicians in primary health care in that country have antibiotic preference for the cephalosporin group. ${ }^{14}$ Antibiotic use is influenced by the personal preference of hospital physicians, limited experience, availability of antibiotics, and the potential effects of marketing by pharmaceutical industries. ${ }^{22}$ The use of higher-generation of cephalosporin and carbapenem by the physicians may occur due to less empirical evidence on antibiotic use provided by WHO before 2013 .

Our study findings suggest that $72.5 \%$ of patients received treatment before hospitalization. Among them, $46.3 \%$ received antibiotics prior to hospitalization, which is consistent with a previous study conducted in Bangladesh (44\%). ${ }^{3}$ According to our study, $94.6 \%$ of antibiotics used for first-line treatment before hospitalization were cefixime, azithromycin, ceftriaxone, ciprofloxacin, ceftaxidime plus amikacin, cefradine, cefaclor, meropenem, cefuroxime, and ceftriaxone plus flucoxacillin other than ampicillin plus gentamicin, and amoxicillin. In our study, we observed that $100 \%$ patients, diagnosed as severe or very severe pneumonia, received injectable antibiotics and among them 98.7\% patients received ceftriaxone, cefotaxime plus amikacin, cefuroxime, ceftaxidime plus amikacin, ceftriaxone plus amikacin, meropenem, cefotaxime, cefepime, ceftriaxone plus gentamicin, ceftazidime, and cefotaxime plus gentamicin as first-line treatment other than a combination of ampicillin plus gentamicin. These antibiotics prescribed by qualified physicians or drug sellers prior to hospitalization may have an influence on antibiotic practices in the inpatient department of hospitals after admission.

In our study, we followed the WHO Pocket Book (2005) ${ }^{16}$ to identify adherence of the hospital's physicians to diagnose, 
classify, and treat pneumonia. All enrolled patients were diagnosed with pneumonia by the hospital physicians. The hospital physicians did not classify pneumonia according to the WHO guidelines, which is crucial for appropriate treatment. Following the WHO Pocket Book (2005), ${ }^{16}$ the study physician diagnosed $54 \%$ patients as having severe pneumonia and $46 \%$ as having very severe pneumonia. We observed that all the patients were treated similarly with antibiotics without any classification of pneumonia or prior investigations like blood cultures and sensitivity tests. To properly treat patients and prescribe antibiotics, physicians should define the illness and severity of illness with clinical judgment and microbiological evidence. This may in turn also reduce the cost of treatment.

According to WHO Pocket Book (2005), ${ }^{16}$ severe pneumonia should be treated with oral amoxicillin and very severe pneumonia with parenteral ampicillin or benzylpenicillin and gentamicin as first-line treatment and accordingly ceftriaxone should be used as a second-line treatment in case of failure of the first-line treatment. In the revised WHO classification, pneumonia was defined as having fast breathing and/or chest indrawing, which needs to be treated at home with oral amoxicillin as first-line treatment (azithromycin as second-line treatment in case of first-line treatment failure), and "severe pneumonia" was defined as having any general danger sign with or without fast breathing which requires referral to hospitals for injectable therapy (ampicillin plus gentamicin as first-line treatment and ceftriaxone as second-line treatment in case of first-line treatment failure). ${ }^{8}$ In our study, among the suggested antibiotics by $\mathrm{WHO}$, amoxicillin and ampicillin plus gentamicin were used in 5\% of cases irrespective of diagnosis before hospitalization, and ampicillin plus gentamicin was used only in 3\% cases of very severe pneumonia $(n=37)$ after hospitalization. This revealed very low adherence to pneumonia treatment guidelines. However, Afreen and Rahman ${ }^{13}$ showed low (3\%) adherence to treatment guidelines including antibiotics and other drugs in pneumonia among the pediatric age group patients in a medical university in Bangladesh. Furthermore, our study reported the use of amikacin among children under 5 years of age, which is something noteworthy for physicians. Aminoglycosides such as amikacin should be used cautiously as it may have clinical toxicities like ototoxicity or nephrotoxicity. ${ }^{23}$

We also found associations of antibiotic use with the age of the patient, respiratory auscultation findings, and chief complaints of the patients. A study conducted in India showed nonassociation of antibiotic practice with comorbidities and association with symptoms of illness, which supports our study findings. ${ }^{24}$

\section{Limitations of the study}

This study was conducted within a short period of time during winter. For this reason, we were not able to identify seasonal variations in antibiotic practice. The study was conducted in a private pediatric teaching hospital in Dhaka city, the scenario of which may differ from a government hospital where physicians are most likely to have undergone IMCI training in accordance with WHO guidelines. Hence, the study findings might be generalized for private hospitals only where pediatric patients are treated.

\section{Conclusion}

The results of our data suggest that the use of injectable antibiotics was high in the private hospital which did not follow the WHO standard treatment guidelines. The observation emphasizes the importance of adherence to high-quality evidence based on recent WHO classification and treatment in accordance with the severity of pneumonia. Enforcing regulatory policy is the need of the hour, which may help to increase the rational use of antibiotics in treating hospitalized children with pneumonia and consequently may prevent increased antimicrobial resistance. However, more researches are sine qua non to identify the seasonal variations of antibiotic practice on the basis of their blood culture and sensitivity both in private and government hospitals following the WHO Pocket Book (2013).

\section{Acknowledgment}

We acknowledge gratefully James P Grant School of Public Health and MPH Program, the hospital authority, for its support and cooperation. We are grateful to all of the study participants and their parents or caregivers for their patience and for sharing information. We are also thankful to our research assistant Aklima Tasrin for her contribution in data collection. International Centre for Diarrhoeal Disease Research, Bangladesh (icddr,b) also gratefully acknowledges the following donors who provided unrestricted support: Government of the People's Republic of Bangladesh, Global Affairs Canada, Swedish International Development Cooperation Agency, and the Department for International Development. The research study was funded by James P Grant School of Public Health, BRAC University, Bangladesh for partial accomplishment of Master of Public Health. 


\section{Disclosure}

The authors report no conflicts of interest in this work.

\section{References}

1. UNICEF. Committing to Child Survival: A Promise RenewedProgress Report 2014. New York, NY: United Nations Children's Fund; 2015. Available from: http://www.unicef.org/publications/files/ APR_2015_9_Sep_15.pdf. Accessed July 31, 2017.

2. Walker CLF, Rudan I, Liu L, et al. Global burden of childhood pneumonia and diarrhoea. Lancet. 2013;381(9875):1405-1416.

3. Naheed A, Saha SK, Breiman RF, et al; Pneumococcal Study Group. Multihospital surveillance of pneumonia burden among children aged $<5$ years hospitalized for pneumonia in Bangladesh. Clin Infect Dis. 2009;48 (Suppl 2):S82-S89.

4. Kiplagat A, Musto R, Mwizamholya D, Morona D. Factors influencing the implementation of integrated management of childhood illness (IMCI) by healthcare workers at public health centers \& dispensaries in Mwanza, Tanzania. BMC Public Health. 2014;14(1):277.

5. Lange S, Mwisongo A, Mæstad O. Why don't clinicians adhere more consistently to guidelines for the Integrated Management of Childhood Illness (IMCI)? Soc Sci Med. 2014;104:56-63.

6. Mulaudzi MC. Adherence to case management guidelines of Integrated Management of Childhood Illness (IMCI) by healthcare workers in Tshwane, South Africa. SAJCH South African J Child Heal. 2015;9(3):89-92. Doi:10.7196/SAJCH.7959.

7. Senn N, Rarau P, Salib M, et al. Use of antibiotics within the IMCI guidelines in outpatient settings in Papua New Guinean children: an observational and effectiveness study. PLoS One. 2014;9(3): e90990.

8. WHO Pocket Book. Pocket Book of Hospital Care for Children: Guideline for the Management of Common Childhood Illness. 2nd ed.; 2013. Available from: http://apps.who.int/iris/bitstream/10665/81170/ 1/9789241548373_eng.pdf?ua=1. Accessed July 31, 2017.

9. Rashid A, Chowdhury A, Rahman SHZ, Begum SA, Muazzam N. Infections by pseudomonas aeruginosa and antibiotic resistance pattern of the isolates from Dhaka Medical College Hospital. Bangladesh J Med Microbiol. 2007;1(2):48-51.

10. Biswas M, Roy D, Tajmim A, et al. Prescription antibiotics for outpatients in Bangladesh: a cross-sectional health survey conducted in three cities. Ann Clin Microbiol Antimicrob. 2014;13:15.

11. NIPORT, Associates M and, International I. Bangladesh Demographic and Health Survey; 2013. Available from: https://dhsprogram.com/pubs/ pdf/fr265/fr265.pdf. Accessed July 31, 2017.
12. WHO. Antimicrobial Resistance: Global Report on Surveillance. Geneva: World Health Organization; 2014. Available from: http:// apps.who.int/iris/bitstream/10665/112642/1/9789241564748_eng.pdf. Accessed July 31, 2017.

13. Afreen S, Rahman MS. Adherence to treatment guidelines in a university hospital: Exploration of facts and factors. Bangladesh J Pharmacol. 2014;9(2):182-188.

14. Hasan SMR, Hossain MM, Akter R, et al. Pattern of antibiotics use at the primary health care level of Bangladesh: survey report-1. Stamford J Pharm Sci. 2009;2(1):1-7.

15. Fahad BM, Matin A, Shill MC, Asish KD. Antibiotic usage at a primary health care unit in Bangladesh. Australas Med J. 2010;3(7):414-421.

16. WHO Pocket Book. Pocket Book of Hospital Care for Children. Geneva: World Health organization; 2005. Available from: http://whqlibdoc. who.int/publications/2005/9241546700.pdf. Accessed July 31, 2017.

17. WHO. What is overweight and obesity? [webpage on the Internet] Geneva: World Health Organization; 2012. Available from: http://www. who.int/dietphysicalactivity/childhood_what/en/index.html. Accessed July 31, 2017.

18. WHO. Weight-for-age BOYS. Child growth standards. Geneva: World Health Organization; 2012. Available from: http://www.who.int/ childgrowth/standards/WFA_boys_0_5_zscores.pdf. Accessed July 31, 2017.

19. WHO. Weight-for-age GIRLS. Child growth standards. Geneva: World Health Organization; 2012. Available from: http://www.who. int/childgrowth/standards/WFA_girls_0_5_zscores.pdf. Accessed July 31, 2017.

20. Chowdhury AK, Rahman SM, Faroque AB, Hasan GA, Raihan SZ. Excessive use of avoidable therapeutic injections in the upazilla health complexes of Bangladesh. Mymensingh Med J. 2008;17(2):S59-S64.

21. Akter SF, Heller RD, Smith AJ, Milly AF. Impact of a training intervention on use of antimicrobials in teaching hospitals. J infect Dev Ctries. 2009;3(6):447-451.

22. Akter SF, Rani MFA, Rahman JA, et al. Antimicrobial use and factors influencing prescribing in medical wards of a tertiary care hospital in Malaysia. Int J Sci Environ Technol. 2012;1(4):274-284.

23. Avent ML, Rogers BA, Cheng AC, Paterson DL. Current use of aminoglycosides: Indications, pharmacokinetics and monitoring for toxicity. Intern Med J. 2011;41(6):441-449.

24. S KI, Chandy SJ, Jeyaseelan L, Kumar R, Suresh S. Antimicrobial prescription patterns for common acute infections in some rural \& urban health facilities of India. Indian J Med Res. 2008;128(2):165-171.
Patient Preference and Adherence

\section{Publish your work in this journal}

Patient Preference and Adherence is an international, peer-reviewed, open access journal that focuses on the growing importance of patient preference and adherence throughout the therapeutic continuum. Patient satisfaction, acceptability, quality of life, compliance, persistence and their role in developing new therapeutic modalities and compounds to optimize

\section{Dovepress}

clinical outcomes for existing disease states are major areas of interest for the journal. This journal has been accepted for indexing on PubMed Central. The manuscript management system is completely online and includes a very quick and fair peer-review system, which is all easy to use. Visit http://www. dovepress.com/testimonials.php to read real quotes from published authors. 